\title{
Viscous Core Liposomes Increase siRNA Encapsulation and Provides Gene Inhibition When Slightly Positively Charged
}

\author{
Shayan Ahmed ${ }^{1}$, Hugo Salmon ${ }^{2}{ }^{(D}$, Nicholas Distasio ${ }^{2}{ }^{\circledR}$, Hai Doan Do ${ }^{1}$, Daniel Scherman ${ }^{1}$, Khair Alhareth ${ }^{1}{ }^{1}$, \\ Maryam Tabrizian ${ }^{2}$ and Nathalie Mignet ${ }^{1, *(D)}$ \\ 1 Unité des Technologies Chimiques et Biologiques Pour la Santé (UTCBS), CNRS, INSERM, \\ Université de Paris, F-75006 Paris, France; shayan.ahmed@etu.parisdescartes.fr (S.A.); \\ hai_doan.do@etu.parisdescartes.fr (H.D.D.); daniel.scherman@parisdescartes.fr (D.S.); \\ khairallah.alhareth@parisdescartes.fr (K.A.) \\ 2 Biomedical Engineering Department and Faculty of Dentistry, McGill University, 3775 University St, \\ Montreal, QC H3A 2B4, Canada; hugo.salmon@parisdescartes.fr (H.S.); \\ nicholas.distasio@mail.mcgill.ca (N.D.); maryam.tabrizian@mcgill.ca (M.T.) \\ * Correspondence: author: nathalie.mignet@parisdescartes.fr
}

\section{check for} updates

Citation: Ahmed, S.; Salmon, H.; Distasio, N.; Do, H.D.; Scherman, D.; Alhareth, K.; Tabrizian, M.; Mignet, N. Viscous Core Liposomes Increase siRNA Encapsulation and Provides Gene Inhibition When Slightly Positively Charged. Pharmaceutics 2021, 13, 479. https://doi.org/ 10.3390/pharmaceutics13040479

Academic Editors: María Luisa Moyá and Manuel López-López

Received: 10 March 2021

Accepted: 26 March 2021

Published: 1 April 2021

Publisher's Note: MDPI stays neutral with regard to jurisdictional claims in published maps and institutional affiliations.

Copyright: (c) 2021 by the authors. Licensee MDPI, Basel, Switzerland. This article is an open access article distributed under the terms and conditions of the Creative Commons Attribution (CC BY) license (https:// creativecommons.org/licenses/by/ $4.0 /)$.

\begin{abstract}
Since its discovery, evidence that siRNA was able to act as an RNA interference effector, led to its acceptation as a novel medicine. The siRNA approach is very effective, due to its catalytic mechanism, but still the limitations of its cellular delivery should be addressed. One promising form of non-viral gene delivery system is liposomes. The variable and versatile nature of the lipids keeps the possibility to upgrade the liposomal structure, which makes them suitable for encapsulation and delivery of drugs. However, to avoid the limitation of fast release for the hydrophilic drug, we previously designed viscous core liposomes. We aimed in this work to evaluate if these viscous core liposomes (NvcLs) could be of interest for siRNA encapsulation. Then, we sought to add a limited amount of positive charges to provide cell interaction and transfection. Cationic lipid dimyristoylaminopropylaminopropyl or the polymer poly(ethylenimine) were incorporated in NvcL to produce positively charged viscous core liposomes (PvcL) by a customized microfluidic device. We found that NvcLs increased the encapsulation efficiency and loading content with regards to the neutral liposome. Both PvcLPEI and PvcLDMAPAP exhibited transfection and GFP knock-down $(\approx 40 \%)$ in both $2 \mathrm{D}$ and $3 \mathrm{D}$ cell cultures. Finally, the addition of slight positive charges did not induce cell toxicity.
\end{abstract}

Keywords: siRNA; neutral viscous core liposomes; positively charged viscous core liposomes; microfluidic device; 3D cell cultures; encapsulation efficiency; loading content; gene delivery; dimyristoylaminopropylaminopropyl; poly(ethylenimine)

\section{Introduction}

Viral vectors constitute the majority of gene delivery vehicles in the clinic [1,2]. Despite the fact that synthetic non-viral vectors are potentially less costly to produce and eventually safer, their transfection ability often remains a limitation to effective gene delivery [3]. The major goal for an increasing number of studies has thus been to render non-viral vectors more efficient and to understand their limitations such as biodistribution, cell internalization, nucleic acid release or nuclear delivery [4,5]. The discovery of siRNA and improvement in mRNA production and in vivo use have re-invigorated the field, as their site of action is in the cytoplasm, which significantly reduces the main limitation of nuclear passage needed for DNA therapeutics. This led to the success of the first liposome-based siRNA therapy used for treating polyneuropathy associated with hereditary transthyretinmediated amyloidosis (hATTR), which was approved by the FDA in 2018 [6]. Another important limitation addressed by hATTR formulation is the lipid chosen. The majority of non-viral vectors which are already described in the literature are positively charged for 
gene delivery including, liposomes, polymers, nanocrystals, inorganic materials, micelles, and proteins in order to facilitate their interaction with anionic nucleic acids and to provide cell interaction and internalization, which will lead to better transfection efficiency [7-9]. However, this cationic charge can impair their circulation time, intracellular release efficiency, and cause toxicity [10-12]. The titratable lipids developed more recently were shown to produce significantly long-circulating lipoplexes with reduced toxicity and nucleic acid delivery at a lower $\mathrm{pH}$ [13].

After several attempts to reduce the charges of lipoplexes [14-16], we sought to formulate lipidic self-assembled nanoparticles with a viscous internal polymeric core, herein referred to as neutral viscous core liposomes (NvcLs). Thickened core liposomes have been proposed before [17]. These studies showed that these liposomes exhibit improved stability and higher encapsulation rate compatible with drug delivery. Moreover, the intertangled polymers forming the liposome core induced a delayed drug delivery [17]. This approach for producing NvcLs nanoparticles with low polydispersity and their thorough characterization of the poloxamer polymer amount within the liposomal core, is the only one reported so far in the literature [18]. These hybrid nanoparticles were prepared with FDA-approved phospholipids and poloxamer to reduce the risk of toxicity and improve their potential for clinical translation. We have shown that small molecules such as rhodamine and doxorubicin could be encapsulated into this novel type of viscous core liposomes with an efficiency of 75\% [18-20]. To expand the application potential of these thickened core liposomes to the widely growing field of RNAi therapeutics, in this paper, we evaluated the potential of neutral viscous core liposomes (NvcLs) to encapsulate siRNA. SiRNA was encapsulated through a home-made ethanolic injection microfluidic device adapted from our previous study, which allowed us to obtain hybrid lipid polymer nanoparticles [18]. Then, in order to provide cell interaction and transfection, we sought to insert a minimal amount of cationic charges to avoid toxicity. We have developed two strategies to provide the necessary cationic charge to the formulation. The first strategy consisted of providing low cationic charges to the lipidic part of the liposome by the addition of 2-\{3-[bis(3-aminopropyl) amino] propylamino\}-N-ditetradecyl-carbamoyl methyl acetamide or dimyristoylaminopropylaminopropyl (DMAPAP) as the cationic lipid [3], and the second was to add a low amount of cationic polymer poly(ethylenimine) to the polymer part of the formulation, entrapped in the core of the liposomes [21,22]. Both lipid (DMAPAP) and polymer (PEI) have been shown to allow the internalization of nucleic acid into the cells, leading to high transfection efficiency $[23,24]$. Herein, we report the entrapment of siRNA in neutral viscous core liposomes (NvcLs) and the transfection efficiency of slightly positively charged viscous core liposomes (PvcLs) in a 2D cell culture, and a first proof of concept in a 3D custom-made microchip. Finally, we addressed the cytotoxicity of the PvcL formulations on endothelial cells.

\section{Materials and Methods}

\subsection{Materials}

DOPC (1,2 dioleoyl-sn-glycero-3-phosphocholine) was from Avanti polar lipids (Alabaster, AL, USA), poloxamer 407 (P407) was provided by BASF (Ludwigshafen, Germany), and branched polyethylenimine, PEI was provided by Sigma-Aldrich (St. Louis, MO, USA). The cationic lipid DMAPAP (2-\{3-[Bis-(3-amino-propyl)-amino]-propylamino\}$\mathrm{N}$-ditetradecyl carbamoyl methyl-acetamide or dimyristoylaminopropylaminopropyl) was synthesized as described [3]. Chloroform and ethanol were purchased from Carlo-Erba reagents (Val-de-Reuil, France). Ultrafiltration units NANOSEP 300k were from PALL filtron (Rehoboth, MA, USA), polyether ether ketone (PEEK) tubes and T-shaped connectors and ferrules were from IDEX Health and Science (Oak Harbor, WA, USA), microfluidic chips were made out of Flexdym $150 \times 150 \mathrm{~mm}^{2}$ sheets (Eden Microfluidics, Paris, France). Endothelial cell growth medium 2 and trypsin/EDTA were obtained from Cedarlane Labs (Burlington, ON, Canada). The medium DMEM/F12 was from Thermo Fisher Scientific (Waltham, MA, USA), recombinant murine TNF-alpha from PeproTech 
(Neuilly-Sur-Seine, France), fetal bovine serum albumin, Embryomax ${ }^{\circledR} 0.1 \%$ Gelatin Solution, and Penicillin-Streptomycin from Sigma-Aldrich (St. Louis, MO, USA). The EA.hy926 (ATCC ${ }^{\circledR}$ CRL2922 ${ }^{\mathrm{TM}}$ ) cell line was purchased from the American Type Culture Collection (ATCC, Manassas, VA, USA).

2.2. Preparation of Conventional Neutral Liposomes, Neutral Viscous Core Liposomes, Positively Charged LIPOSOMES, and Positively Charged Viscous Core Nanocarriers with PEI or DMAPAP and SiRNA

The conventional neutral liposomes (NL) and neutral viscous core liposomes (NvcL) were prepared by the same procedure as described previously [18]. The positively charged liposomes (PLPEI/DMAPAP) and cationic hybrid liposome/poloxamer (PvcL PEI/DMAPAP) with PEI and DMAPAP were also prepared with the same method as NL and NvcL, only with the addition of positively charged moieties in the composition. The PLs were prepared by the ethanolic injection method using a syringe pump apparatus (Harvard apparatus PHD 2000). One syringe (1 mL) was filled with an ethanolic solution of DOPC $(20 \mathrm{mg} / \mathrm{mL}, 25.4 \mu \mathrm{mol} / \mathrm{mL})$ or with DMAPAP and DOPC with a ratio 1:9 $(2 \mathrm{mg} / \mathrm{mL}$ DMAPAP and $18 \mathrm{mg} / \mathrm{mL}$ DOPC), and another one $(10 \mathrm{~mL})$ with Milli-Q ${ }^{\circledR}$ water containing siRNA $(30 \mathrm{nM})$ and PEI $(1.3 \%$ or $30 \mu \mathrm{g} / \mathrm{mL})$ or only Milli-Q ${ }^{\circledR}$ water containing siRNA $(30 \mathrm{nM})$. Both syringes were fixed on the syringe pump apparatus and the injection speed was set at $0.5 \mathrm{~mL} / \mathrm{min}$ for the lipid solution and $5 \mathrm{~mL} / \mathrm{min}$ for the aqueous solution. The apparatus ran for $1 \mathrm{~min}$ giving a final volume of $5.5 \mathrm{~mL}$, followed by ethanol evaporation that yielded a final concentration of DOPC $(1.8 \mathrm{mg} / \mathrm{mL})$ and DMAPAP $(0.2 \mathrm{mg} / \mathrm{mL}$ or $10 \%$ of the total lipid content) after the ethanol evaporation. PvcLs were prepared using the same procedure as PLs but the aqueous phase was replaced by the poloxamer 407 (P407) solution $(5 \% w / v$ or $50 \mathrm{mg} / \mathrm{mL})$ containing siRNA $(30 \mathrm{nM})$ and PEI $(0.06 \%$ or $30 \mu \mathrm{g} / \mathrm{mL})$ (ratio PEI/poloxamer) or only siRNA $(30 \mathrm{nM})$, which flowed into the system with a rate of $5 \mathrm{~mL} / \mathrm{min}$. The graphical representation and composition of each formulation is available in Table 1.

Table 1. Graphical representation of tested formulations and their composition. The siRNA concentration was $30 \mathrm{nM}$ for all the formulations

\begin{tabular}{|c|c|c|c|c|c|c|}
\hline $\begin{array}{c}\text { Poloxamer } \\
\text { PEI }{ }^{+} \\
\text {siRNA }=\end{array}$ & NL & $\begin{array}{l}\text { NvcL } \\
(\infty)+\infty \\
(\infty)\end{array}$ & $\begin{array}{r}\text { PLPeI } \\
\left(\begin{array}{l}+\frac{7}{+}+ \\
+\frac{7}{7}\end{array}\right.\end{array}$ & 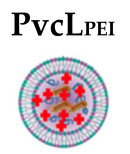 & $\begin{array}{l}\text { PLDMAPAP } \\
\text { (a) }\end{array}$ & PvcLdMAPAP \\
\hline DOPC $(\mathrm{mg} / \mathrm{mL})$ & 2 & 2 & 2 & 2 & 1.8 & 1.8 \\
\hline Poloxamer $(\mathrm{mg} / \mathrm{mL})$ & - & 50 & - & 50 & - & 50 \\
\hline DMAPAP $(\mathrm{mg} / \mathrm{mL})$ & - & - & - & - & 0.2 & 0.2 \\
\hline PEI $(\mathrm{mg} / \mathrm{mL})$ & - & - & 0.03 & 0.03 & - & - \\
\hline
\end{tabular}

\subsection{Transmission Electron Microscopy}

The NL and NvcL formulations were observed by transmission electron microscopy (TEM). Briefly, $10 \mu \mathrm{L}$ of the sample was deposited on a carbon-coated copper grid, the excess solution was removed after $2 \mathrm{~min}$ by a filter paper. This was followed by the application of $5 \mu \mathrm{L}$ of uranyl acetate $1 \%$ to the same grid for $2 \mathrm{~min}$, the excess stain was then removed with a filter paper. The sample is subsequently air-dried at room temperature. The grid placed on a slide was inserted in the microscope. The sample analysis was performed with a JEOL JEM $100 \mathrm{~S}$ (JEOL Ltd., Tokyo, Japan) TEM operating at $80 \mathrm{kV}$. TEM images were captured using an Orius Sc 200 digital camera (Gatan-Roper Scientific, Evry, France).

\subsection{Dynamic Light Scattering (DLS)}

A Malvern Zetasizer Nanoseries Nano ZS (Malvern Instruments, France) was used to determine the mean particle hydrodynamic diameter ( $\mathrm{Z}$ average), polydispersity index (PDI), and zeta potential. The neutral and cationic formulation diameter and PDI were 
determined at $25^{\circ} \mathrm{C}$ by quasi-elastic light scattering. The scattering was measured at a fixed angle of $173^{\circ}$ and positioned at 4.65. As for the zeta potential measurements, a dilution of $1 / 50$ of each formulation was carried out in $150 \mathrm{mM} \mathrm{NaCl}$ prior to themeasurements.

\subsection{Nanoparticle Tracking Analysis}

Nanoparticle tracking analysis (NTA) was conducted with a $640 \mathrm{~nm}$ laser at $t=25{ }^{\circ} \mathrm{C}$ (NanoSight 300, Amesbury, UK) to measure the size of PvcLs nanoparticles. Each formulation was diluted by 1/500 in Milli-Q and the measurements were performed in a dynamic flow mode controlled by a built-in syringe pump at level 60 . The particle size is reported as the hydrodynamic diameter, determined by following the 2D trajectory of each particle over a tracking time with the NanoSight NTA 3.4 software (NanoSight 300, Amesbury, UK).

\subsection{Ultrafiltration by Centrifugation}

Ultrafiltration columns (Pall Filtron Europe, Postmouth, UK) with 300 kDa molecular weight cut-offs were used to separate free poloxamer and non-encapsulated siRNA in the filtrate from the nanoparticles. The filtration was carried out by centrifugation at $13,000 \mathrm{rpm}$ for $30 \mathrm{~min}$ at $4{ }^{\circ} \mathrm{C}$ in a refrigerated bench-top centrifuge (Hettich Rotanta $460 \mathrm{RF}$, Tuttlingen, Germany).

\subsection{Ribo Green Assay}

The $100 \mu \mathrm{g} / \mathrm{mL}$ of siRNA suspension was diluted 50-folds with a Tris-EDTA (TE) buffer to reach a concentration of $2 \mu \mathrm{g} / \mathrm{mL}$. The series of dilutions $(1,0.5,0.1$, and $0.02 \mu \mathrm{g} / \mathrm{mL}$ ) was prepared and $100 \mu \mathrm{L}$ of each concentration was transferred to a 96-well plate. A total of $100 \mu \mathrm{L}$ of diluted RiboGreen dye (1:200 dilutions in TE buffer) was added to each siRNA concentration. Fluorescence measurements for the calibration curve were made after 5 min of incubation at ambient temperature using a TECAN instrument at emission wavelength of $525 \mathrm{~nm}$. The quantification of encapsulated siRNA was then carried out using the same procedure discussed above for the calibration curve. After filtration, the siRNA in the filtrate of each sample was quantified and expressed as \% using equations.

The following are the loading content and encapsulation efficiency equations:

Loading content $(\mathrm{LC} \%)=(($ total siRNA - free siRNA $) /($ amount of lipid component $)) \times 100$;

Encapsulation efficiency $(\mathrm{EE} \%)=(($ total siRNA - free siRNA $) /($ total siRNA $)) \times 100$.

\subsection{Cytotoxicity Assay}

The immortalized endothelial EA.hy926 cell line, commonly used in various angiogenesis and cancer studies, was chosen for in vitro cytotoxicity assays as they can be transfected more easily than primary endothelial cells [25,26].

Briefly, the cells were grown in DMEM containing $2 \mathrm{mM} \mathrm{L-glutamine,} 100 \mathrm{U} / \mathrm{mL}$ penicillin and $100 \mathrm{\mu g} / \mathrm{mL}$ streptomycin, $10 \%$ bovine serum at $37^{\circ} \mathrm{C}$, and under $5 \% \mathrm{CO}_{2}$. Cells were plated onto 96 -well plates at 20,000 cells per well, containing $100 \mu \mathrm{L}$ of culture medium. Twenty-four hours after plating, $100 \mu \mathrm{L}$ of medium containing the nanoparticles of interest (final concentrations ranging from 0.06 to $2 \mathrm{mg} / \mathrm{mL}$ of DOPC, $1.5-50 \mathrm{mg} / \mathrm{mL}$ of P407, 0.9375 to $30 \mu \mathrm{g} / \mathrm{mL}$ of PEI, and 0.00625 to $0.2 \mathrm{mg} / \mathrm{mL}$ of DMAPAP) was added to the each predefined well. After $24 \mathrm{~h}$ of exposure, the cell viability was evaluated using the MTT test in a microplate reader and recorded the absorbance at $560 \mathrm{~nm}$ (Infinite F200 Pro-TECAN). All measurements for different formulations were performed in triplicate for three independent experiments.

The cell viability equation:

Viability $\%=($ Asample - mean $($ Ablank $)) /($ mean (Asolvent $)-$ mean $($ Ablank $)) \times 100 \%$ 


\subsection{Cell Culture, Fixation, Imaging, and GFP Quantification}

Each well of a 24 -well microplate was seeded at 64,000 cells $/ \mathrm{mL}$ with mouse primary endothelial cells suspended in the endothelial growth medium (EGM-2). After 2-3 days in culture and at the confluency, the cells were transfected with various formulations of siRNA for $4 \mathrm{~h}$. The cells were then rinsed twice with PBS, were fixed using paraformaldehyde $4 \%$, and were stained with DAPI for nucleus and phalloidin-i594 for actin.

Triple fluorescence measurements were performed on an Eclipse TE2000. Acquisition was performed using the open-source Micromanager 1.4, while the image analysis and quantification of GFP level were achieved using the open-source Fiji ImagJ-win64 and a custom-made script. All the conditions were performed in triplicate for each two sets of experiments. The GFP expression of the cells was quantified by taking the same images as analyzed for the qualitative analysis. The GFP expression was calculated with the help of the Fiji imageJ-win64 software for the confocal images by taking into account the number of cells, while taking each nucleus (DAPI) into consideration. The images were first segmented to isolate the structure of interest and the images were then transformed into binary ones. Finally, the nuclei of the stained cells were selected and the various parameters, such as counts and areas, were determined. These results ensure that the qualitative and quantitative analysis have shown a similar kind of result.

\subsection{Endothelium-on-Chip 3D Cell Culture, Fixation, Imaging, and GFP Quantification}

For the endothelium-on-chip device fabrication, the soft thermoplastic foils were hotembossed at $170{ }^{\circ} \mathrm{C}$ on a metallic mold, which was obtained by welding gauge 25 needles. After demolding, the chip is closed on a thin thermoplastic layer and interfaced using thermoplastic studs. A permanent bonding is achieved by baking the chips at $80^{\circ} \mathrm{C}$ for $30 \mathrm{~min}$ [27]. The chips are subsequently sterilized flowing $70 \% v / v$ ethyl alcohol diluted in distilled water, rinsed with Milli-Q water, and then coated with $0.1 \% w / v$ gelatin as an extracellular matrix in an incubator at $37^{\circ} \mathrm{C}$ for $30 \mathrm{~min}$, and were seeded with cells as described in Section 2.9. The chips were flipped several times to ensure a full coverage of the V-shape tubular structure of the device with a monolayer of endothelial cells. The confluent cell treatment with RNA formulations and cell fixation procedure for the fluorescence microscopy analyses were performed as described for the 2D cell culture in Section 2.9.

\section{Results and Discussion}

Despite the possibility to encapsulate hydrophilic drugs into a liposome core, the applications are rather limited since the equilibrium between the internal and external aqueous media leads to a fast exchange of the hydrophilic drug. This is why, in the last two years, liposomes with increased viscosity into the aqueous core have been designed to enhance the retention of hydrophilic drugs. This current study is the continuation of this recent research work in terms of formulating neutral liposomes (NLs) and hybrid formulation named neutral viscous core liposomes (NvcLs) as a carrier. The idea of replacing the aqueous core with the viscous core was to improve the encapsulation efficiency of the drug in the carrier. We chose to incorporate poloxamer, which is a FDA approved triblock copolymer, into the core. Previous investigations on viscous core liposomes showed a feasible controlled release, as drug encapsulation is enhanced thanks to the viscosity of the core and the drug release requires first the dissolution of the polymeric network $[17,18,28]$. Moreover, we proposed an automated mixing device to form homogeneous liposomes with the viscous core. As with conventional methods, highly polydispersed liposomes would have formed. This customized microfluidic device was used to mix the microfluidic technology and the ethanolic injection process and produce the formulations shown in Figure 1. Two syringes, containing an ethanolic solution of lipids, and an aqueous or polymeric solution with or without siRNA, respectively were fixed on the microfluidic device and pushed together by the help of a push syringe, as described in Section 2.2 (Figure 1). All the parameters regarding the length of tubing system, diameter of tubing 
system, injection speed, ratio of lipid and polymer, and their concentrations were studied in detail in our previous publication [18].

In this study, we aimed to adapt the process to encapsulate the siRNA and to form hybrid liposomes encapsulating siRNA in order to answer the following questions: (1) Is it possible to entrap siRNA in neutral viscous core liposomes? (2) Is the device adapted to form positively charged viscous core liposomes? (3) Do slightly positively charged viscous core liposomes allow transfection without inducing cellular toxicity?

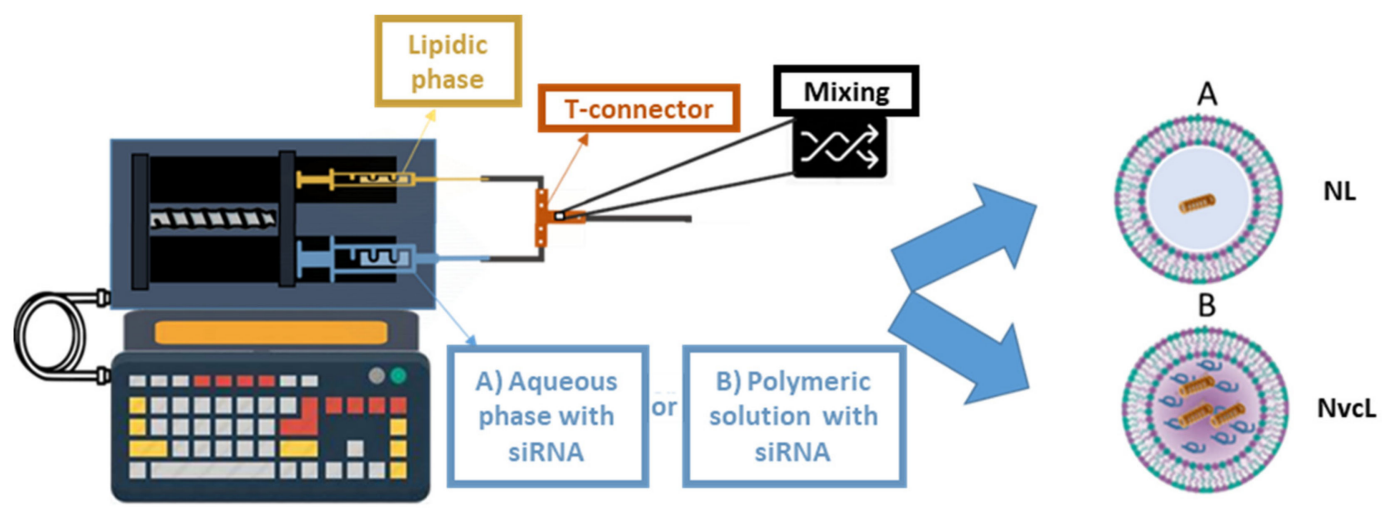

Figure 1. Graphical representation of push syringe technique for the preparation of (A). Neutral Liposomes (NLs) and (B). Neutral viscous core Liposomes (NvcLs).

\subsection{Size and Charge Characterization of Neutral Formulations and Their siRNA Encapsulation} Efficiency and Loading Content

The first question addressed in this paper was whether increasing the viscosity of the liposome core could entrap siRNA in neutral liposomes. Using the device displayed in Figure 1 and described in Material and Methods, siRNA was added during the process, within the aqueous phase to force its encapsulation during liposome formation. The TEM images showed similar sizes and morphologies for both nanoparticles (Figure 2). The large non-spherical objects in Figure 2a can be the aggregated lipids which fused with each other by drying the TEM grid. Figure $2 b$ shows the NvcLs which are better in terms of their morphologies and sizes, may be due to the stability provided by their inner core. Further detailed characterization with DLS measurements confirmed a similar size for NL and NvcL as shown in Table 2, and a better PDI for NvcL consistent with TEM observations and our previous results [18]. Overall, DLS analyses showed nanoparticles with a PDI of $0.18-0.23$ and an average size ranging from of 100 to $125 \mathrm{~nm}$. The particle size distribution curves by the intensity of NL + siRNA and NvcL + siRNA is presented in Figure S2.

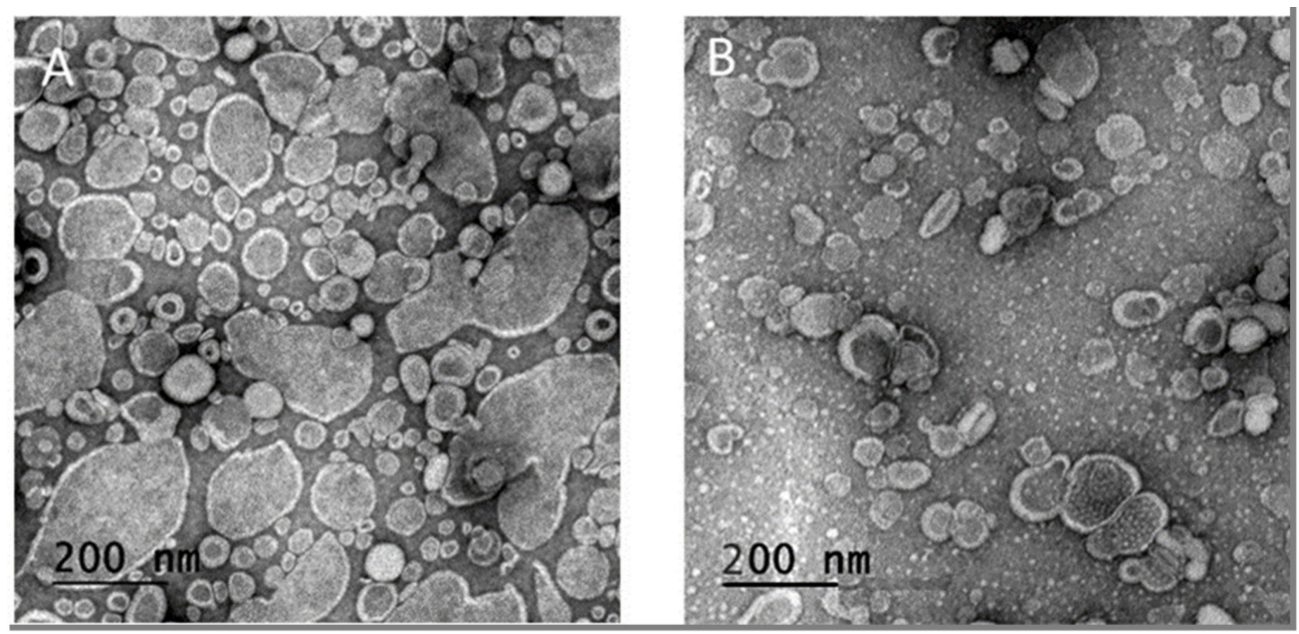

Figure 2. TEM images of neutral formulations. (A) NL and (B) NvcL formulations. 
To demonstrate that the thicker core of NvcL could improve the encapsulation of siRNA, the free siRNA was eliminated by ultrafiltration and then quantified using the RiboGreen assay. Equations (1) and (2), given in Material and Methods, was then applied and indicated that an encapsulation efficiency (\%) and loading content (\%) of NL were $8.45 \pm 0.21$ and $0.016 \pm 0.009$ and for NvcL $96.7 \pm 0.9$ and $0.144 \pm 0.003$, respectively as shown in Table 2. A higher loading content was quantified with NvcL in comparison with conventional liposomes, which we attributed to the viscosity introduced by poloxamer in the self-assembled nanoparticles. To ensure that the encapsulation yield was not artificially increased by the presence of poloxamer within the filtrate, a calibration curve of siRNA was created in the presence of poloxamer and used for the quantification (Figure S1 in Supplementary Data). Furthermore, we quantified the whole passage of free poloxamer by the Baleux assay to ensure that poloxamer would not clog the filter [18].

This is the first example of viscous core liposomes shown to entrap small nucleic acid fragments. In general, nanogels such as the thiol-conjugated hyaluronic acid and disulfide cross-linked dextrin or chitosan particles have been used to encapsulate siRNA or DNA in nanogels. These nanogels obtained by ionic gelation allow an efficient encapsulation of nucleic acids $[10,29]$.

Table 2. Characterization of NL and NvcL formulations. Dynamic light scattering (DLS) measurements for size are represented by the mean hydrodynamic average diameter by intensity and the polydispersity index (PDI). This table also presents the encapsulation efficiency $\%$ and loading content $\%$ of siRNA encapsulated in NL and NvcL. The values and standard deviation are given for nine values, triplicates measured three times each $(n=3)$.

\begin{tabular}{ccccc}
\hline & Size (nm) & PDI & E.E\% & L.C\% \\
\hline NL & $104 \pm 20$ & $0.23 \pm 0.03$ & $8.45 \pm 0.21$ & $0.02 \pm 0.009$ \\
NvcL & $124 \pm 15$ & $0.18 \pm 0.04$ & $96.7 \pm 0.88$ & $0.14 \pm 0.003$ \\
\hline
\end{tabular}

\subsection{Size and Charge Characterization of Positively Charged Formulations (PvcL)}

Nevertheless, in order to obtain liposomes able to interact with cells, we sought to render the liposomes which are slightly positively charged. As positive charges are used to interact with all surfaces, we wondered if the microfluidic device made with push syringe and T-shaped tubing, made with poly-ether-ether-ketone (PEEK) which is highly resistive and inert, could appropriately allow forming PvcLs. In order to carry out this study the positive charges were added to NL and NvcL formulations via two approaches, either by the addition of PEI (Figure 3a) within the poloxamer polymer phase or by the addition of the cationic DMAPAP lipid (Figure $3 b$ ) to the lipidic membrane. The cationic DMAPAP lipid carries ionizable amines on a polyamine moiety which had initially been developed in our laboratory [23]. Both PEI and the DMAPAP lipid have been extensively studied and have been reported to improve the encapsulation efficiency and internalization of nucleic acid into cells [3,21,30-33]. Using the ethanolic injection and microfluidic device for mixing, the lipids were dissolved in an ethanol solution and the polymers in the aqueous one as shown in Figure 4, according to the amount indicated in Table 1.

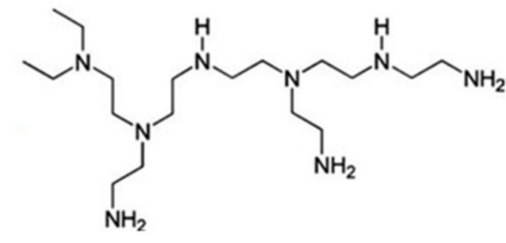

(a)

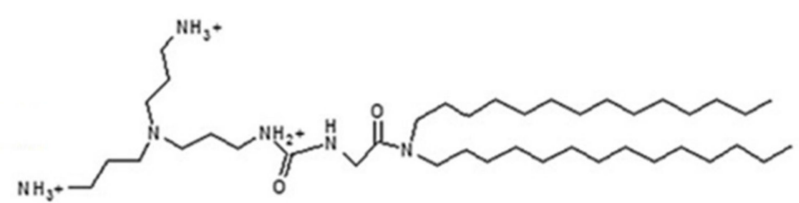

(b)

Figure 3. (a) Structure of polyethylenimine (branched) and (b) structure of dimyristoylaminopropylaminopropyl (DMAPAP) lipid. 


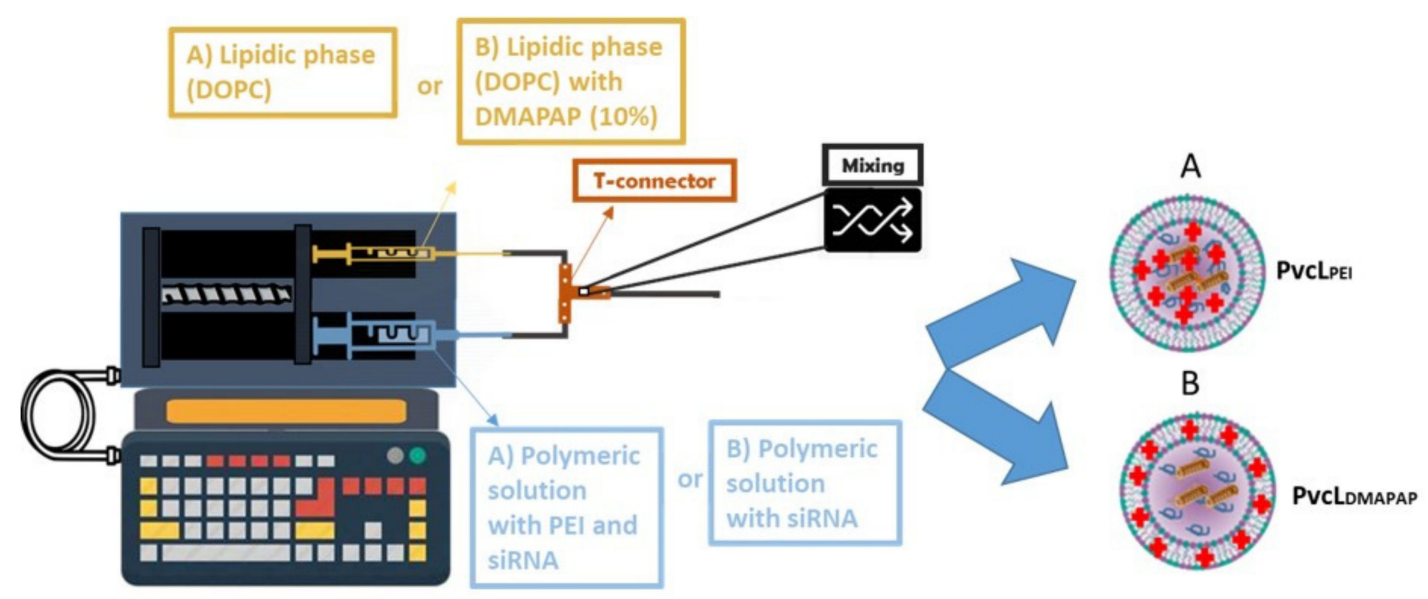

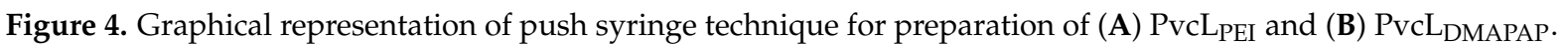

In the case of PvcL DMAPAP, we added $10 \% w / w$ of DMAPAP lipid to the neutral lipid, representing only $0.4 \% w / w$ with regards to the total lipid + polymer weight, in the formulation which is quite lower than what is usually described in the literature where cationic liposomes contain a higher percentage of cationic lipids (approx.: 30-50\%) [34-37].

We used two techniques for size measurement analyses, DLS which measures variations in scattering intensity from a bulk sample, and NTA which measures particle-byparticle mobility related to Brownian motion [38]. In our case both techniques indicated quite monodispersed nanoparticles with a PDI of 0.16-0.22 and a size ranging from 100 to $180 \mathrm{~nm}$ typically used for nano-formulations, thereby confirming the suitability of microfluidic method for producing positively charged liposomes (PLs) and positively charged viscous core liposomes (PvcLs) with positively charged moieties such as DMAPAP or PEI (Table 3). The low PDI of PvcL formulations may be due to the presence of poloxamer, which stabilizes and provides a defined shape and size to the liposomes [18]. DLS was also used to measure the electrophoretic mobility and determine the surface charge. As expected, PvcLPEI and PvcL $\mathrm{DMAPAP}_{\text {P }}$ exhibited positive zeta with a value of $+8.9 \pm 1.8 \mathrm{mV}$ and $+5.9 \pm 0.9 \mathrm{mV}$, respectively, whereas the zeta potential was $+5.8 \pm 1.7 \mathrm{mV}$ for PLPEI and $+6.9 \pm 1.8 \mathrm{mV}$ for PL DMAPAP (Table 1). These low positive charges are expected to reduce the toxicity without compromising the transfection efficiency, as in literature the high positively charged nanoparticles were reported to cause toxicity issues [7,12,35].

Table 3. DLS and nanoparticle tracking analysis (NTA) analyses for the PLPEI, PvcL PEI $_{\text {PL }}$ DMAPAP, and PvcL DMAPAP formulations. The size is represented by the mean hydrodynamic average diameter by intensity and the polydispersity index (PDI). The values and standard deviation are given for nine values, triplicates measured three times each $(n=3)$.

\begin{tabular}{ccccc}
\hline & \multicolumn{4}{c}{ NTA } \\
\cline { 2 - 5 } & Size $\mathbf{( n m )}$ & PDI & Size $(\mathbf{n m})$ & Zeta Potential (mV) \\
\hline PL & $97 \pm 9$ & $0.34 \pm 0.02$ & $180 \pm 4.3$ & $+5.8 \pm 1.7$ \\
PVCLE & $143 \pm 12$ & $0.17 \pm 0.05$ & $143 \pm 1.7$ & $+8.9 \pm 1.8$ \\
PL $_{\text {DMAPAP }}$ & $113 \pm 10$ & $0.25 \pm 0.06$ & $172 \pm 1.3$ & $+6.9 \pm 1.8$ \\
PVCL $_{\text {DMAPAP }}$ & $122 \pm 16$ & $0.16 \pm 0.02$ & $174 \pm 2.7$ & $+5.9 \pm 0.9$ \\
\hline
\end{tabular}

\subsection{SiRNA Cell Transfection}

\subsubsection{Cell Transfection in Microplates}

The third question we had asked was whether these low positive charged liposomes could be able to deliver their siRNA content into endothelial cells and transfect those cells. The transfection of siRNA was performed in vitro on GFP expressing murine endothelial cells. Confocal microscopy imaging analyses showed the GFP downregula- 
tion for cells treated with PLPEI + siRNA, PVcLPEI + siRNA, PLDMAPAP + siRNA, and PvcL $_{\text {DMAPAP }}+$ siRNA, when compared with non-treated cells (Figure $5 a, b$ ). No changes were observed within the actin filament network, nuclei or cell densities, suggesting that the treatments had no obvious adverse effects on these cells. These results provide a qualitative analysis of gene knockdown efficiency and low cytotoxicity of siRNA encapsulated cationic formulations.

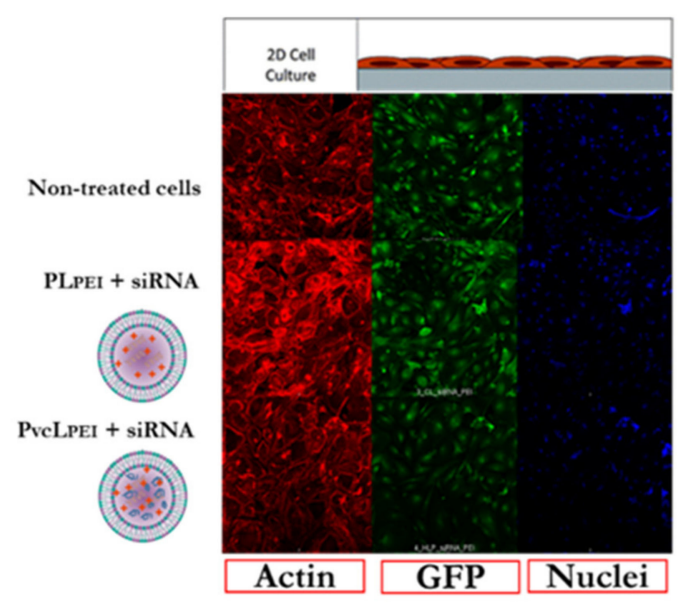

(a)

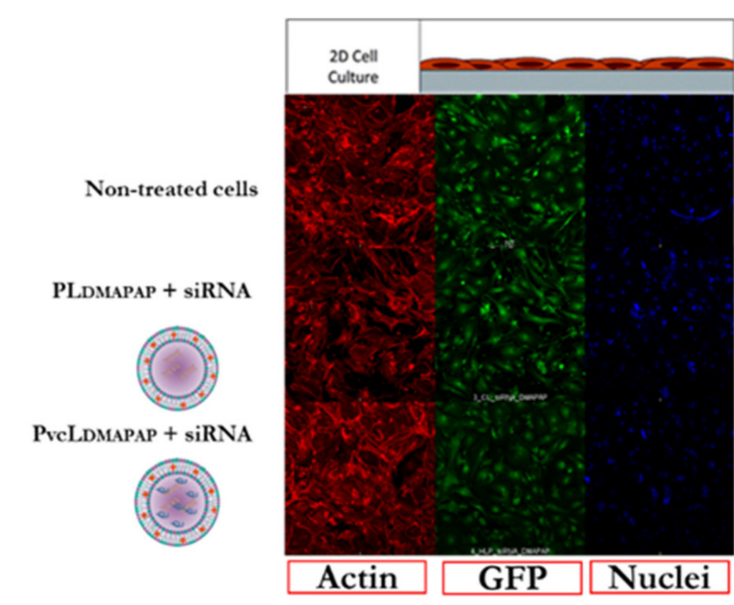

(b)

Figure 5. Confocal imaging of endothelial cells treated with various formulations in vitro. (a) Non-treated PLPEI and PvcL $_{\text {PEI }}$; (b) non-treated PL DMAPAP and PvcL DMAPAP treated cells.

To further solidify the deduction from the results of confocal images, the quantification analysis was carried out using the software imageJ. The graph (a) in Figure 6 presents the cells treated with positively charged polymer-based formulations; PLPEI + siRNA and PvcL $_{\text {PEI }}+$ siRNA in comparison with non-treated cells and PEI + siRNA as a control. The graph (b) in Figure 6 presents the cells treated with positively charged lipid-based formulations; PL $_{\text {DMAPAP }}+$ siRNA and PvcL DMAPAP + siRNA in comparison with nontreated and lipofectamine + siRNA taken as a positive control.

Starting from PEI, the liposomes containing PEI taken as a control did show only 90\% of GFP inhibition, which was expected for formulations containing only 1.3\% PEI. Similarly, the liposomes containing DMAPAP taken as a control showed no inhibition of GFP expression, which was consistent with the fact that only $10 \% w / w$ of DMAPAP was incorporated into the formulation. Lipidic nanoparticles are less efficient to internalize siRNA into the cells and provide transfection efficiency lower than polymers. This is why other hybrid formulations containing positively charged lipids and anionic polymers were also developed, providing higher stability and transfection ability [39-41].

One can see, in Figure 6, that both PvcLPEI + siRNA and PvcL DMAPAP + siRNA were the more efficient formulations tested. They downregulate up to 55\% of the GFP expression when compared with non-treated and controls. To note, this inhibition level is obtained for $<1 \% w / w$ positive charge for PVCLPEI and PvcLDMAPAP. Indeed, the amount of positive content in the viscous core liposomes is 25 times less in weight due to the amount of incorporated poloxamer within the core. This is important to note as most of the very efficient formulations, described in the literature, present a high positive content, but these cationic liposomes also lead to toxicity issues. Several laboratories have tried to reduce the toxicity by managing the compositions and ratios of the liposomes, some of the examples are DOTAP with carboxymethyl- $\beta$-cyclodextrin, DOTAP/Chol/DOPE, and DOTAP/DOPE $[35,36]$. 
PEI

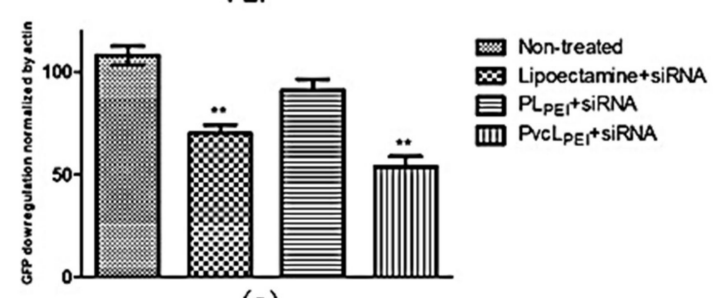

(a)

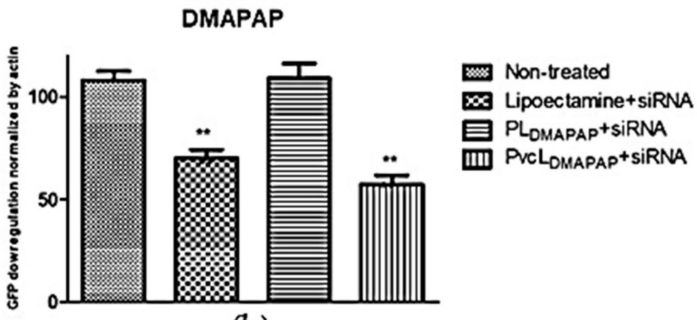

(b)

Figure 6. Quantitative analysis of GFP downregulation (normalize by actin) with siRNA encapsulated in cationic formulations. (a) Non-treated lipofectamine, PLPEI, and PvcLPEI, (b) non-treated lipofectamine, PL DMAPAP, and PvcL $_{\text {DMAPAP }}$ treated cells. Student's $t$-test was applied, $p<0.05\left(^{* *}\right)$ for PvcL PEI, lipofectamine, and PvcL DMAPAP formulations $(n=3)$.

\subsubsection{Cell Transfection Inside the Microchip}

In parallel to cell transfection in microplates, we designed a 3D cell culture model in a microchip to test the transfection efficiency of formulations for further applications such as drug development, as 3D models better mimic the natural cell physiological environment [42].

The gene inhibition study was carried out on the same GFP expressing endothelial cells and the same number of cells inside the 3D microchip. Actin and DAPI immunostaining of cells confirmed the formation of a tubular monolayer but their signals varied between different treatments as the 3D nature of the vessels made it more difficult to directly compare fluorescence levels between the samples. This issue made the quantification of GFP expression more difficult. However, the confocal microscopy imaging analyses showed a GFP downregulation for the cells treated with PLPEI + siRNA, PvcLPEI + siRNA, PL DMAPAP + siRNA, and PvcL DMAPAP + siRNA, when compared with non-treated cells (Figure $7 \mathrm{a}, \mathrm{b}$ ). These results should be further confirmed, and the system improved, but these preliminary results are in accordance with the cell transfection obtained in the 2D culture. Interestingly, the 3D cell culture used a very little amount of formulation and medium $(\sim 7 \mu \mathrm{L})$ representing only $7 \%$ of the amount used for cell transfection in 96-well microplates $(\sim 100 \mu \mathrm{L})$. Therefore, it is highly appropriate for precious samples such as siRNA or mRNA, for instance, and should be further developed.

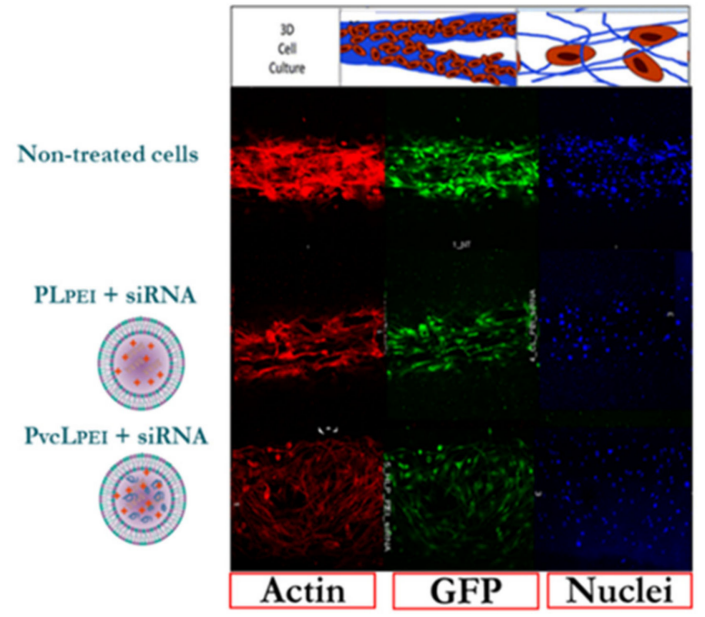

(a)

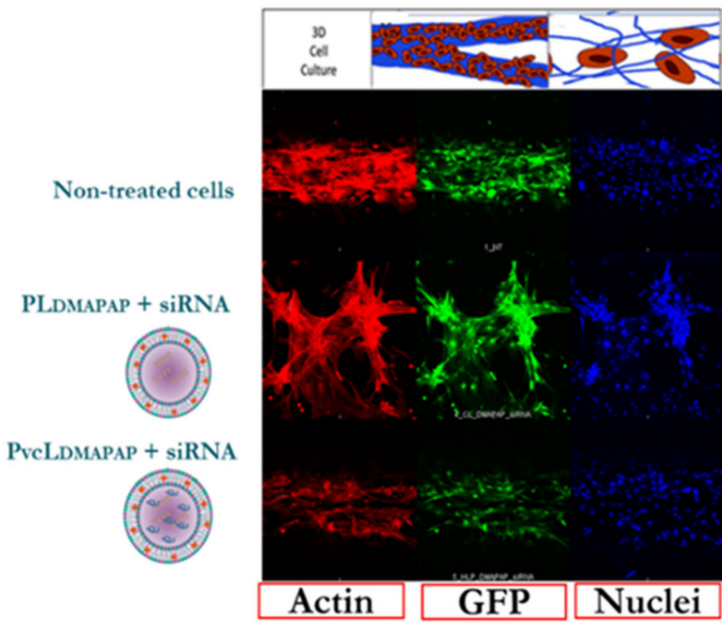

(b)

Figure 7. Confocal imaging of endothelial cells in the three-dimensional (3D) culture microchip treated with various formulations in vitro, (a) non-treated PLPEI and PvcLPEI; (b) non-treated PL DMAPAP and PvcL DMAPAP treated cells.

\subsection{Cytotoxicity Assay}

Cytotoxicities of the positively charged liposome (PL) and positively charged viscous core liposome (PvcL) formulations with PEI or DMAPAP were evaluated on EA.hy926 
cells using the MTT cell viability test. The viability results for PL and PvcL formulations are presented in Figure 8 as a function of lipid (A), P407 (B), PEI (C), and DMAPAP concentrations (D), present in the formulations. The mean inhibition concentration 50 (IC50) values determined for each curve is shown in Table 3. In EA.hy926 cells, the IC50 value was found to be approximately $2 \mathrm{mg} / \mathrm{mL}$ for DOPC, $52 \mathrm{mg} / \mathrm{mL}$ for P $407,0.03 \mathrm{mg} / \mathrm{mL}$ for PEI, and $0.21 \mathrm{mg} / \mathrm{mL}$ for DMAPAP, when these moieties were in the PL and PvcL formulations. The PvcLDMAPAP did not show any toxicity at the evaluated concentrations so in this case the IC50 values are not applicable.

The cytotoxicity results obtained for positively charged formulations were similar for PLPEI, PvcLPEI, and PLDMAPAP when considering DOPC or poloxamer concentrations (Table 4). Noteworthy, the PvcL made with DMAPAP did not show any toxicity in the concentration range studied. The IC50 values were well above the concentrations used in the in vitro experiments. These results indicated no cytotoxic effect within the concentration studied for the transfection evaluation, as the formulations of Table 1 were further diluted into half when added to the culture medium used for cell transfection. These results further highlight their biocompatibility at these concentrations of lipid, poloxamer, PEI, and DMAPAP.

Table 4. The mean inhibition concentration 50 (IC50) values for EA.hy926 cells as a function of lipid, P407, PEI, DMAPAP, and siRNA content in the nanocarrier formulations (N/A: Not applicable).

\begin{tabular}{cccccc}
\hline Cell Lines & Component & PL $_{\text {PEI }}$ & PvcL $_{\text {PEI }}$ & PL $_{\text {DMAPAP }}$ & PvcL $_{\text {DMAPAP }}$ \\
\hline \multirow{3}{*}{$\begin{array}{c}\text { Endothelial Cells } \\
\text { (IC50) }\end{array}$} & Lipid $(\mathrm{mg} / \mathrm{mL})$ & 2 & $>2$ & $>2$ & $>5$ \\
\cline { 2 - 6 } & $\mathrm{P} 407(\mathrm{mg} / \mathrm{mL})$ & $\mathrm{N} / \mathrm{A}$ & 52 & $\mathrm{~N} / \mathrm{A}$ & $>100$ \\
\cline { 2 - 6 } & $\mathrm{PEI}(\mathrm{mg} / \mathrm{mL})$ & 0.03 & 0.03 & $\mathrm{~N} / \mathrm{A}$ & $\mathrm{N} / \mathrm{A}$ \\
\cline { 2 - 6 } & $\mathrm{DMAPAP}(\mathrm{mg} / \mathrm{mL})$ & $\mathrm{N} / \mathrm{A}$ & $\mathrm{N} / \mathrm{A}$ & 0.21 & $>1$ \\
\hline
\end{tabular}

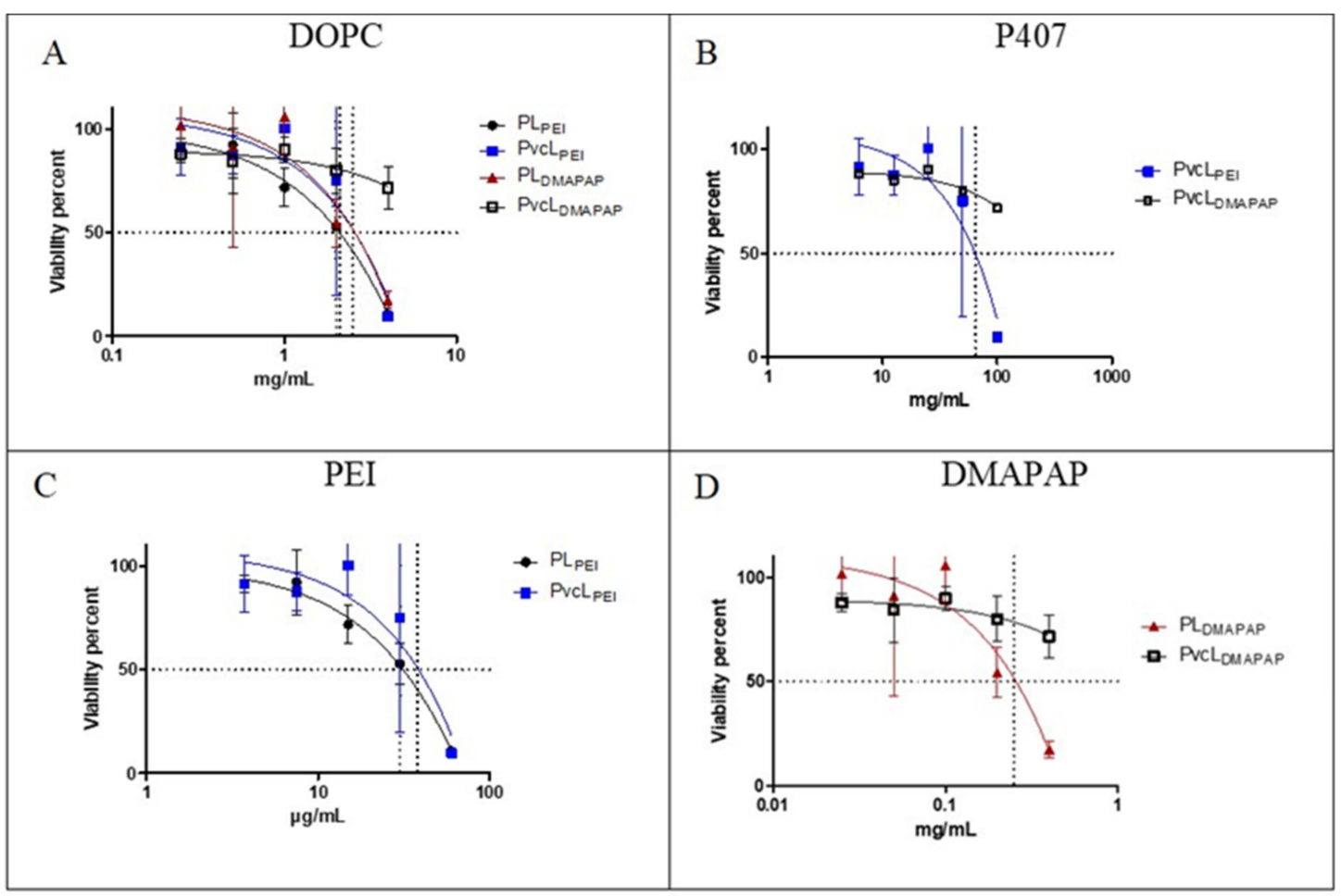

Figure 8. (A) DOPC, (B) P407, (C) PEI, and (D) DMAPAP concentration effects on EA.hy926 cell viability. Gaussian nonlinear regression fit and two-way ANOVA was applied $p<0.05(n=3)$. 


\section{Conclusions}

The present results displayed that the NvcL formulation has better encapsulation efficiency (\%) than NL due to the viscous core of liposomes. The addition of slightly positive charges to formulations encapsulating siRNA have efficiently inhibited GFP expression in endothelial cells constitutively expressing GFP. Thus, these PvcLs formulations are able to facilitate siRNA cell internalization without inducing significant toxicity, while releasing the siRNA inside the cells. The DLS and NTA measurements suggest that the optimal size and PDI for the formulations and formulation concentrations are compatible for in vivo use and should be evaluated in the future. The cell culture in the microchip allowed transfecting and imaging cells in a 3D configuration, which is considered a more relevant physiologically environment for investigating various diseases. The $3 \mathrm{D}$ cell culture model showed preliminary results consistent with those obtained with the 2D culture, while minimizing the consumption of reagents and medium. Finally, this work highlights the potential of lipid/polymer nanoparticles as a vector for siRNA delivery and potentially other nucleotide-based drugs such as mRNA, for either therapeutic or vaccination purposes. This work suggests the significance of advanced technology such as microfluidics and $3 \mathrm{D}$ models for the preparation and the evaluation of innovative therapeutic strategies, including self-assembled nanoparticles.

Supplementary Materials: The following are available online at https:/ / www.mdpi.com/article/10. 3390/pharmaceutics13040479/s1, Figure S1: Calibration curves for siRNA quantification at different concentrations, (a) siRNA in the absence of P407 and (b) siRNA in the presence of P407. Figure S2: Particle size distribution curves by intensity, (a) NL + siRNA and (b) NvcL + siRNA.

Author Contributions: Conceptualization, methodology, software, validation, formal analysis, investigation, data curation, writing original draft preparation, visualization, S.A.; methodology, software, formal analysis, investigation, writing-review and editing, H.S.; methodology, software, investigation, writing - review and editing, N.D.; formal analysis, investigation, H.D.D.; resources, writing-review and editing, D.S.; writing-review and editing, visualization, K.A.; methodology, resources, writing-review and editing, M.T.; conceptualization, methodology, formal analysis, resources, data curation, writing - review and editing, visualization, supervision, N.M. All authors have read and agreed to the published version of the manuscript.

Funding: This research was funded by the France Canada Research Fund (FFCR) for this project and the Higher Education Commission of Pakistan for awarding PhD funding to Shayan AHMED.

Institutional Review Board Statement: Not applicable.

Informed Consent Statement: Not applicable.

Data Availability Statement: Not applicable.

Acknowledgments: The authors thank Hélène Dhotel for the DMAPAP synthesis, Fond France Canada pour la Recherche (FFCR) for funding staff exchanges, the Higher Education Commission of Pakistan for providing Shayan Ahmed's scholarship for PhD, the TEM platform of the Universite de Paris, UMS3612 CNRS, US25 Inserm, P-MIM, and BASF (Ludwigshafen, Germany) for providing Poloxamer 407.

Conflicts of Interest: The authors declare no conflict of interest.

\section{References}

1. Hacein-Bey-Abina, S.; Hauer, J.; Lim, A.; Picard, C.; Wang, G.P.; Berry, C.C.; Martinache, C.; Rieux-Laucat, F.; Latour, S.; Belohradsky, B.H.; et al. Efficacy of Gene Therapy for X-Linked Severe Combined Immunodeficiency. N. Engl. J. Med. 2010, 363, 355-364. [CrossRef]

2. Metzner, C.; Dangerfield, J. Surface Modification of Retroviral Vectors for Gene Therapy. Viral Gene Ther. 2011. [CrossRef]

3. Tranchant, I.; Thompson, B.; Nicolazzi, C.; Mignet, N.; Scherman, D. Physicochemical optimisation of plasmid delivery by cationic lipids. J. Gene Med. 2004, 6, S24-S35. [CrossRef]

4. Escriou, V.; Mignet, N.; Miller, A. Auto-associative lipid-based systems for non-viral nucleic acid delivery. In Introduction to Systems Biology; World Scientific Publishing: Singapore, 2019; pp. 237-270. 
5. Kostarelos, K.; Miller, A.D. What role can chemistry play in cationic liposome-based gene therapy research today? Genet. Genom. Fish Phenomics 2005, 53, 71-118.

6. Wahlich, J.; Desai, A.; Greco, F.; Hill, K.; Jones, A.T.; Mrsny, R.J.; Pasut, G.; Perrie, Y.; Seib, F.P.; Seymour, L.W.; et al. Nanomedicines for the Delivery of Biologics. Pharmaceutics 2019, 11, 210. [CrossRef] [PubMed]

7. Barua, S.; Ramos, J.; Potta, T.; Taylor, D.; Huang, H.-C.; Montanez, G.; Rege, K. Discovery of cationic polymers for non-viral gene delivery using combinatorial approaches. Comb. Chem. High Throughput Screen. 2011, 14, 908-924. [CrossRef]

8. Zhu, L.; Mahato, R.I. Lipid and polymeric carrier-mediated nucleic acid delivery. Expert Opin. Drug Deliv. 2010, 7, 1209-1226. [CrossRef]

9. Gao, L.; Ma, N. DNA-Templated Semiconductor Nanocrystal Growth for Controlled DNA Packing and Gene Delivery. ACS Nano 2011, 6, 689-695. [CrossRef]

10. Ahmed, S.; Alhareth, K.; Mignet, N. Advancement in nanogel formulations provides controlled drug release. Int. J. Pharm. 2020, 584, 119435. [CrossRef]

11. Do, H.D.; Couillaud, B.M.; Doan, B.-T.; Corvis, Y.; Mignet, N. Advances on non-invasive physically triggered nucleic acid delivery from nanocarriers. Adv. Drug Deliv. Rev. 2019, 138, 3-17. [CrossRef] [PubMed]

12. Lv, H.; Zhang, S.; Wang, B.; Cui, S.; Yan, J. Toxicity of cationic lipids and cationic polymers in gene delivery. J. Control. Release 2006, 114, 100-109. [CrossRef]

13. Buck, J.; Grossen, P.; Cullis, P.R.; Huwyler, J.; Witzigmann, D. Lipid-Based DNA Therapeutics: Hallmarks of Non-Viral Gene Delivery. ACS Nano 2019, 13, 3754-3782. [CrossRef]

14. Breton, M.; Leblond, J.; Tranchant, I.; Scherman, D.; Bessodes, M.; Herscovici, J.; Mignet, N. Lipothioureas as Lipids for Gene Transfection: A Review. Pharmaceuticals 2011, 4, 1381-1399. [CrossRef]

15. Thompson, B.; Mignet, N.; Hofland, H.; Lamons, D.; Seguin, J.; Nicolazzi, C.; De La Figuera, N.; Kuen, R.L.; Meng, X.Y.; Scherman, D.; et al. Neutral Postgrafted Colloidal Particles for Gene Delivery. Bioconjug. Chem. 2005, 16, 608-614. [CrossRef]

16. Nicolazzi, C.; Mignet, N.; De La Figuera, N.; Cadet, M.; Ibad, R.T.; Seguin, J.; Scherman, D.; Bessodes, M. Anionic polyethyleneglycol lipids added to cationic lipoplexes increase their plasmatic circulation time. J. Control. Release 2003, 88, 429-443. [CrossRef]

17. Zhang, B.; Chen, J.; Lu, Y.; Qi, J.; Wu, W. Liposomes interiorly thickened with thermosensitive nanogels as novel drug delivery systems. Int. J. Pharm. 2013, 455, 276-284. [CrossRef]

18. Ahmed, S.; Corvis, Y.; Gahoual, R.; Euan, A.; Lai-Kuen, R.; Couillaud, B.M.; Seguin, J.; Alhareth, K.; Mignet, N. Conception of nanosized hybrid liposome/poloxamer particles to thicken the interior core of liposomes and delay hydrophilic drug delivery. Int. J. Pharm. 2019, 567, 118488. [CrossRef]

19. Xiong, M.P.; Wang, Y.; Tu, S. Liposome-Encapsulated Hydrogels for Use in a Drug Delivery System. US Patent 14/116,919, 8 May 2014.

20. Wang, Y.; Tu, S.; Pinchuk, A.N.; Xiong, M.P. Active drug encapsulation and release kinetics from hydrogel-in-liposome nanoparticles. J. Colloid Interface Sci. 2013, 406, 247-255. [CrossRef]

21. Höbel, S.; Aigner, A. Polyethylenimine (PEI)/siRNA-Mediated Gene Knockdown In Vitro and In Vivo. Adv. Struct. Saf. Stud. 2010, 623, 283-297. [CrossRef]

22. Nimesh, S. Polyethylenimine as a promising vector for targeted siRNA delivery. Curr. Clin. Pharmacol. 2012, 7, 121-130. [CrossRef]

23. Byk, G.; Dubertret, C.; Escriou, V.; Frederic, M.; Jaslin, G.; Rangara, R.; Pitard, B.; Crouzet, J.; Wils, P.; Schwartz, B.; et al. Synthesis, Activity, and Structure-Activity Relationship Studies of Novel Cationic Lipids for DNA Transfer. J. Med. Chem. 1998, 41, 224-235. [CrossRef]

24. Tang, M.X.; Szoka, F.C. The influence of polymer structure on the interactions of cationic polymers with DNA and morphology of the resulting complexes. Gene Ther. 1997, 4, 823-832. [CrossRef]

25. Bouïs, D.; Hospers, G.A.; Meijer, C.; Molema, G.; Mulder, N.H. Endothelium in vitro: A review of human vascular endothelial cell lines for blood vessel-related research. Angiogenesis 2001, 4, 91-102. [CrossRef] [PubMed]

26. Gresch, O.; Altrogge, L. Transfection of Difficult-to-Transfect Primary Mammalian Cells. Methods Mol. Biol. 2011, 801, 65-74. [CrossRef]

27. Salmon, H.; Rasouli, M.R.; DiStasio, N.; Tabrizian, M. Facile engineering and interfacing of styrenic block copolymers devices for low-cost, multipurpose microfluidic applications. Eng. Rep. 2021, e12361. [CrossRef]

28. Bettini, R.; Colombo, P.; Massimo, G.; Catellani, P.L.; Vitali, T. Swelling and drug release in hydrogel matrices: Polymer viscosity and matrix porosity effects. Eur. J. Pharm. Sci. 1994, 2, 213-219. [CrossRef]

29. Lee, H.; Mok, H.; Lee, S.; Oh, Y.-K.; Park, T.G. Target-specific intracellular delivery of siRNA using degradable hyaluronic acid nanogels. J. Control. Release 2007, 119, 245-252. [CrossRef]

30. Bessodes, M.; Dhotel, H.; Mignet, N. Lipids for Nucleic Acid Delivery: Cationic or Neutral Lipoplexes, Synthesis, and Particle Formation. Methods Mol. Biol. 2019, 123-139. [CrossRef]

31. Boussif, O.; Lezoualc'H, F.; Zanta, M.A.; Mergny, M.D.; Scherman, D.; Demeneix, B.; Behr, J.P. A versatile vector for gene and oligonucleotide transfer into cells in culture and in vivo: Polyethylenimine. Proc. Natl. Acad. Sci. USA 1995, 92, 7297-7301. [CrossRef]

32. Manta, S.; Renault, G.; Delalande, A.; Couture, O.; Lagoutte, I.; Seguin, J.; Lager, F.; Houzé, P.; Midoux, P.; Bessodes, M.; et al. Cationic microbubbles and antibiotic-free miniplasmid for sustained ultrasound-mediated transgene expression in liver. J. Control. Release 2017, 262, 170-181. [CrossRef] 
33. Manta, S.; Delalande, A.; Renault, G.; Bureau, M.F.; Lebertre, M.; Marie, C.; Scherman, D.; Midoux, P.; Mignet, N.; Pichon, C. Positively Charged Microbubbles to Target Nucleic Acid Delivery with Ultrasound. Act. J. RITS 2015. Available online: https:/ / www.hal.inserm.fr/inserm-01154976 (accessed on 10 March 2021).

34. Elsana, H.; Olusanya, T.O.B.; Carr-Wilkinson, J.; Darby, S.; Faheem, A.; Elkordy, A.A. Evaluation of novel cationic gene based liposomes with cyclodextrin prepared by thin film hydration and microfluidic systems. Sci. Rep. 2019, 9, 1-17. [CrossRef]

35. Lechanteur, A.; Sanna, V.; Duchemin, A.; Evrard, B.; Mottet, D.; Piel, G. Cationic Liposomes Carrying siRNA: Impact of Lipid Composition on Physicochemical Properties, Cytotoxicity and Endosomal Escape. Nanomaterials 2018, 8, 270. [CrossRef]

36. Stuart, D.D.; Kao, G.Y.; Allen, T.M. A novel, long-circulating, and functional liposomal formulation of antisense oligodeoxynucleotides targeted against MDR1. Cancer Gene Ther. 2000, 7, 466-475. [CrossRef]

37. Jääskeläinen, I.; Lappalainen, K.; Honkakoski, P.; Urtti, A. Requirements for Delivery of Active Antisense Oligonucleotides into Cells with Lipid Carriers. In Methods in Enzymology; Elsevier: Amsterdam, The Netherlands, 2004; Volume 387, pp. 210-230.

38. Campardelli, R.; Trucillo, P.; Reverchon, E. A Supercritical Fluid-Based Process for the Production of Fluorescein-Loaded Liposomes. Ind. Eng. Chem. Res. 2016, 55, 5359-5365. [CrossRef]

39. Rhinn, H.; Largeau, C.; Bigey, P.; Kuen, R.L.; Richard, M.; Scherman, D.; Escriou, V. How to make siRNA lipoplexes efficient? Add a DNA cargo. Biochim. Biophys. Acta (BBA) Gen. Subj. 2009, 1790, 219-230. [CrossRef]

40. Schlegel, A.; Bigey, P.; Dhotel, H.; Scherman, D.; Escriou, V. Reduced in vitro and in vivo toxicity of siRNA-lipoplexes with addition of polyglutamate. J. Control. Release 2013, 165, 1-8. [CrossRef]

41. Valero, L.; Alhareth, K.; Romero, J.E.; Viricel, W.; Leblond, J.; Chissey, A.; Dhotel, H.; Roques, C.; Arruda, D.C.; Escriou, V.; et al. Liposomes as Gene Delivery Vectors for Human Placental Cells. Molecules 2018, 23, 1085. [CrossRef] [PubMed]

42. Langhans, S.A. Three-Dimensional in Vitro Cell Culture Models in Drug Discovery and Drug Repositioning. Front. Pharmacol. 2018, 9, 6. [CrossRef] [PubMed] 